\title{
Atitudes de gestantes e da população geral quanto aO USO de SUBSTÂNCIAS DURANTE A GESTAÇÃO
}

\author{
Adriana da Matta \\ Lissandra Vieira Soares ${ }^{2}$; Lisiane Bizarro ${ }^{3}$
}

Investigaram-se atitudes permissivas ou restritivas ao uso de substâncias psicoativas (SPA) e medicações sem prescrição, durante a gestação e a amamentação. Os participantes da população geral $(n=172)$ e gestantes $(n=137)$ responderam uma enquete sobre cenários nos quais uma gestante e uma lactante poderiam ou não consumir SPAs e medicações. $\mathrm{O}$ uso de SPAs e medicações foi restringido pela maioria. Dentre os participantes que permitiram o consumo, esse deveria ser em frequência e/ou doses menores. Dentre as gestantes, houve discrepância entre atitudes e comportamento, pois parte das gestantes fumou durante a gestação, mesmo tendo atitude restritiva.

Descritores: Atitude; Gravidez; Lactação; Comportamento Aditivo.

\footnotetext{
${ }^{1}$ Psicóloga, Doutoranda em Psicologia, Instituto de Psicologia, Universidade Federal do Rio Grande do Sul, Porto Alegre, RS, Brasil. E-mail: adriza.ez@terra.com.br.

2 Psicóloga. E-mail: lissandra.soares@yahoo.com.br.

${ }^{3}$ Psicóloga, Doutor em Psicologia, Professor Associado, Instituto de Psicologia, Universidade Federal do Rio Grande do Sul, Porto Alegre, RS, Brasil. E-mail: lisiane.bizarro@ufrgs.br.
} 


\section{AtTitudes Of PREgnant WOMEn AND THE GENERAL POPULATION TOWARDS PSYCHOACTIVE SUBSTANCE USE DURING PREGNANCY}

Permissive and restrictive attitudes concerning the use of psychoactive substances and medication without prescription during pregnancy and breastfeeding were investigated. Participants in the general population $(n=172)$ and pregnant women $(n=137)$ answered a questionnaire about contexts in which a pregnant or breastfeeding woman could use psychoactive substances and medication or not. The majority chose to restrict the use of these substances. Participants who allowed consumption indicated lower frequency or dosage. Among the pregnant women, a discrepancy was found between attitudes and behavior, as part of them smoked during pregnancy, despite their restrictive attitude.

Descriptors: Attitude; Pregnancy; Lactation; Behavior, Addictive.

\section{Actitudes de las embarazadas e de la población general Respecto al USO DE SUSTANCIAS PSICOACTIVAS DURANTE EL EMBARAZO}

Fueron investigadas actitudes de permisión o restricción del uso de sustancias psicoactivas (SPAs) y medicamentos sin prescripción durante el embarazo y la lactancia. Los participantes de la población general $(n=172)$ y embarazadas $(n=137)$ contestaron una encuesta acerca de escenarios en los cuales una embarazada y una lactante podrían o no consumir SPAs y medicaciones. El uso de SPAs y medicamentos fue restringido por la mayoría. Entre los participantes que permitieron el consumo, este debiera ser con frecuencia y/o dosis menores. Entre gestantes, hubo discrepancia entre actitudes y comportamiento, pues parte de ellas fumó durante el embarazo, mismo teniendo actitud restrictiva.

Descriptores: Actitude; Embarazo; Lactancia; Conducta Adictiva.

\section{Introdução}

O uso de substâncias psicoativas (SPA) e de medicações sem indicação profissional, durante a gestação, representa uma área específica na pesquisa sobre a dependência química e merece atenção especial, em razão de suas consequências, que podem ser extremas também para a saúde do feto, a ponto de se manterem presentes durante toda a vida do indivíduo exposto às SPAs ainda no útero ${ }^{(1-2)}$.

Apesar dos conhecidos danos à saúde do feto, a partir de preocupantes dados epidemiológicos, oriundos de estudos consistentes, é possível supor que existe certa permissividade no consumo de SPAs e de medicações sem orientação profissional, durante a gestação e a amamentação. O ambiente em que vivem ou circulam essas gestantes ou mães também pode influenciar seus hábitos, agindo como importante regulador, estimulando, de forma direta ou indireta, ou reprimindo o uso das SPAs ${ }^{(3)}$.

Conhecer o que se pensa, o que se sente e o que se faz a respeito de tal hábito, em termos de conceitos tais como "bom" e "mau", "desejável" e "indesejável", "evitar" ou "buscar" compreende o que se define como atitude. Conceito oriundo do campo da Psicologia Social, consiste no resultado de uma avaliação, que gera uma representação na memória que, por sua vez, afeta a maneira e a forma como se prevê um comportamento ${ }^{(4)}$.

Se, para modificar um comportamento, existe a 
necessidade de se entender as atitudes subjacentes a esse comportamento ${ }^{(4)}$, a fim de melhor planejar ações preventivas efetivas, faz-se necessário conhecer as atitudes tanto na população geral quanto nas gestantes, a respeito do hábito de consumir SPAs, durante a gestação e a amamentação. A gravidez é, de forma típica, período que pode determinar mudanças nos padrões de comportamento e de consumo de SPAs e, assim, é importante se conhecer o que a população geral indica a respeito do uso de SPAs durante a gestação, bem como de que forma isso se aplica às gestantes, se o que elas pensam é coerente com o que entende a população geral e coerente com o que se sabe do ponto de vista médico.

\section{Atitudes relativas ao uso de SPAs durante a gestação}

Embora se defina atitude como a combinação entre crenças, sentimentos e comportamentos, a afirmação de que tais elementos devem ser consistentes entre si é bastante questionável, já que é possível pensar de determinada forma, e nem sempre agir de modo coerente e consistente com essa determinada forma. O quanto uma atitude permite prever o comportamento dependerá das características do indivíduo, da situação e da atitude em si, e sua avaliação não ocorre facilmente. Além disso, a atitude pode variar em função da força das associações do seu objeto na memória ${ }^{(4)}$.

Ainda que uma gestante ou uma lactante considere que não deveria estar usando SPAs, o conceito de atitude permite entender que é possível pensar dessa forma e, ainda assim, fazer uso de SPAs. Ou seja, quando existe uma atitude, a discrepância entre o pensar e o comportarse pode se fazer presente, produto do que se define como ambivalência. Se a atitude depende de diversos fatores, sabe-se que mulheres fumantes ou com parceiros fumantes, residentes em áreas menos favorecidas economicamente, são mais tolerantes ao fumo durante a gestação. Além disso, mulheres fumantes tendem a não perceber os riscos do fumo durante a gestação como particularmente relevantes para si, enquanto entendem a restrição como algo exagerado oriundo de uma sociedade antitabagismo ${ }^{(5)}$. Os conselhos de amigos ou familiares também podem contribuir para a formação das atitudes, especialmente quando a percepção do risco de tal consumo é subestimada pela mulher ${ }^{(3)}$. É possível que o mesmo se aplique a informações obtidas de outras fontes, como campanhas governamentais, internet $\mathrm{e}$ conselhos médicos.

Órgãos públicos ligados às políticas de saúde e organizações não governamentais (ONG), por vezes trabalhando em conjunto, vêm desenvolvendo uma série de campanhas para prevenir e combater o uso de drogas. Tais campanhas podem apresentar caráter combativo, envolvendo intervenções, cujo enfoque é o amedrontamento, o cientificismo, o moralismo ou o apoio, ou caráter positivo, abordagem que se utiliza de estratégias que apostam no fortalecimento do indivíduo e de sua inserção em um grupo ${ }^{(6)}$. Independentemente das críticas aplicáveis a cada abordagem, o que se conclui é que existe esforço desses órgãos e organizações não governamentais em reduzir, combater e prevenir o uso de drogas de forma geral. A população tem acesso a tais campanhas e entende que a gestação deve ser, talvez mais que qualquer outra condição, preservada do uso de SPAs. Uma das substâncias cujo combate é mais evidente é o cigarro.

No Brasil, a partir do início da década de 1990, o Ministério da Saúde, através do Instituto Nacional do Câncer, implementou ações de natureza intersetorial e de abrangência nacional para controlar as determinantes sociais e econômicas da expansão do consumo de tabaco, com o objetivo de reduzir a prevalência de fumantes e a consequente morbimortalidade, o que ocorreu através de estratégias que envolvem a redução da iniciação no tabagismo e o aumento da cessação entre os dependentes ${ }^{(7)}$.

Alguns resultados positivos permitem validar os esforços de tais instituições para a prevenção do uso de SPAs na gestação. Esses autores investigaram o impacto das imagens com advertências sanitárias de embalagens de cigarros, desenvolvidas pelo programa brasileiro de controle do tabaco, utilizando uma tarefa para estudos de emoção e comportamento, comparando as imagens do programa com imagens controle. A imagem que apresentou uma gestante fumando foi considerada muito aversiva mesmo entre os fumantes, sendo que a imagem que apresentava uma mulher (não gestante) fumando não teve o mesmo julgamento ${ }^{(8)}$.

Entre outros efeitos, as campanhas ajudam a disseminar informações a respeito das consequências desse tipo de consumo, atingindo não apenas gestantes, mas seus familiares e sua comunidade. E, se as pessoas entendem que a mensagem de alguma campanha tem alta relevância pessoal, sua atenção à mensagem, bem como a busca por informações a respeito do tópico em questão, tende a crescer. Em um estudo norueguês, foi identificada redução significativa no uso de álcool durante a gestação, bem como mudança nas atitudes em relação ao consumo de álcool, nesse período da vida, em direção à restrição, concomitantes ao desenvolvimento de campanha de prevenção promovida por um órgão nacional ${ }^{(9)}$.

Independentemente de esforços governamentais ou não, as SPAs e medicações sem orientação profissional continuam a ser consumidas por gestantes em índices que podem ser considerados preocupantes. Partindo da importância dos dados relativos ao consumo largamente expostos na literatura, apesar dos conhecidos prejuízos para o desenvolvimento, provocados pelas SPAs na gestação e na amamentação, e considerando que as atitudes frequentemente podem ser antecedentes dos comportamentos $^{(4)}$, o objetivo do presente estudo foi conhecer as atitudes em relação ao consumo de SPAs, durante a gestação e a amamentação, tanto na população geral quanto entre as gestantes, se de restrição ou de permissividade, e, se permissiva, qual a natureza de tal permissividade, se em relação à frequência, à quantidade ou ao tipo de substância ou de uma combinação entre esses. A investigação de tais elementos deve permitir outros desenvolvimentos e embasar consequentes intervenções que possam se mostrar efetivas no incentivo à cessação e à prevenção do tabagismo na gestação. 


\section{Métodos}

A coleta de dados para o presente estudo ocorreu em duas etapas, a primeira delas em 2007, em que uma amostra, oriunda da população geral, foi investigada, e a segunda, em 2008, com amostra composta apenas por gestantes. Nas duas etapas do estudo, a seleção da amostra utilizou critério de conveniência. Os participantes da população geral foram convidados a ingressar no estudo nos mais diversos ambientes, enquanto as gestantes foram convidadas em salas de espera para atendimentos de acompanhamento médico pré-natal, em dois hospitais da rede pública do município de Porto Alegre, RS, que autorizaram a aplicação das enquetes mediante aprovação dos Comitês de Ética em Pesquisa próprios.

\section{Participantes}

A amostra oriunda da população geral foi composta por 172 pessoas entre 15 e 75 anos (média=38; dp=15). São mulheres $54,4 \%$ da amostra, e $31,4 \%$ do total de sujeitos entrevistados concluíram o ensino médio. Quando investigados os hábitos relativos ao consumo de SPAs, revelou-se que $25,6 \%$ da amostra tem por hábito apenas consumir bebidas alcoólicas e 10\% definiram-se como fumantes, unicamente. Dez por cento da amostra bebe e fuma (55,2\% desses são homens). Investigou-se, também, o número de sujeitos que fazem uso de medicações sem prescrição médica, encontrando-se 1,2\%. Não fazem uso de nenhuma substância $46 \%$ da amostra. Do total de sujeitos que revelaram consumir bebidas alcoólicas, $52,3 \%$ são mulheres, bem como, do total de sujeitos fumantes, 58,8\% são mulheres. No entanto, as mulheres são a maioria daqueles que revelaram não utilizar nenhum tipo de SPAs (62,5\%). Alguns participantes oriundos da população geral eram acompanhantes das gestantes em suas consultas de pré-natal.

A amostra relativa à segunda etapa do estudo foi composta por 137 gestantes entre 18 e 43 anos (média $=28,17 ; \mathrm{dp}=6,28$ ). Quanto à escolaridade, 36\% das gestantes apresentaram ensino fundamental incompleto, e $19,1 \%$ apresentaram ensino fundamental completo. Apresentaram o ensino médio completo 30,5\%, enquanto $9,9 \%$ apresentaram ensino superior incompleto, e apenas $3,8 \%$ apresentaram ensino superior completo. Investigouse, também, o trimestre de gestação, sendo que $20,6 \%$ das gestantes se encontravam dentro do primeiro trimestre, $35,3 \%$ no segundo e $44,1 \%$ no terceiro. Os hábitos relativos ao consumo de SPAs foram investigados de duas maneiras, a primeira delas de forma geral e a segunda durante a gestação. Entre as gestantes, e de forma geral, 4,4\% afirmaram consumir bebidas alcoólicas e 7,3\% fumam, enquanto $2,9 \%$ afirmaram tanto fumar quanto consumir bebidas alcoólicas. Durante a gestação, 14,6\% das gestantes afirmaram terem consumido alguma quantidade de bebidas alcoólicas, sendo a cerveja a bebida mais consumida (por 52,6\% das gestantes que revelaram consumir bebidas alcoólicas), sendo que, dessas, a maioria restringiu o consumo a ocasiões especiais $(81,3 \%)$ ou aos domingos (12,5\%). Quando investigado o consumo de cigarros durante a gestação, 17,5\% das gestantes afirmaram tê-los consumido, a metade dessas tendo restringido o consumo a um ou dois cigarros por dia, e 38,9\% dessas consumiram até meia carteira de cigarros por dia.

\section{Instrumento}

O instrumento utilizado para investigar as atitudes em relação ao consumo de SPAs, durante a gestação, na amostra da população geral foi uma enquete autoaplicável que buscou verificar o quão permissivo é o participante quanto ao consumo de bebidas alcoólicas, de cigarros e de outras drogas, durante os períodos de gravidez e de amamentação. Para tanto, foram elaboradas questões que ofereciam alguns cenários. No caso das bebidas alcoólicas, eram apresentados cenários de ocasiões especiais (casamentos, aniversários e comemorações de ano-novo), almoços de domingo e refeições diárias, associados à possibilidade de se consumir bebidas alcoólicas ou não e, em caso de o participante pensar que a gestante poderia fazê-lo em alguma dessas ocasiões (ou em todas), o(s) tipo(s) de bebida(s) e a quantidade. Os mesmos cenários eram colocados para a hipótese de uma mãe estar em fase de amamentação. Também foi investigado se os participantes pensam que uma gestante ou lactante pode consumir cigarros, medicamentos sem prescrição e drogas ilegais e, em caso positivo, com que frequência e em qual quantidade. Foram oferecidas alternativas de respostas que previam que a gestante ou lactante poderia consumir a substância em questão como o fazia costumeiramente antes de engravidar, poderia consumir, desde que em quantidade menor e/ou uma bebida considerada mais "fraca", ou não consumir. O instrumento utilizado para investigar as atitudes em relação ao consumo de SPAs, durante a gestação na amostra de gestantes, era o mesmo apresentado à amostra da população geral, acrescido de questões que investigavam o trimestre de gestação, se a gestante havia consumido bebida alcoólica na presente gestação e, em caso positivo, o tipo de bebida, a quantidade e a frequência. Também foi investigado se as gestantes fumaram ou consumiram medicamentos sem prescrição, na presente gestação.

\section{Resultados}

Os dados foram submetidos à análise de frequências. Foram destacados para a presente seção, os dados que se mostraram significativos, considerados entre si e em relação à literatura científica disponível. Foram analisadas as hipóteses do consumo de bebidas alcoólicas em ocasiões especiais, aos domingos e diariamente, o consumo de cigarros, o uso de medicação sem indicação médica e o uso de drogas ilícitas durante a gestação.

\section{Do consumo de bebidas alcoólicas em ocasiões especiais}

Da amostra oriunda da população geral, 71,8\% dos participantes pensam que uma gestante não poderia consumir bebidas alcoólicas em ocasiões especiais. Dos participantes que pensam que a gestante poderia consumi- 
las, $27,7 \%$ entendem que a gestante pode consumir, desde que em menor quantidade daquela costumeira $(11,8 \%)$ ou bebidas "fracas" $(12,4 \%)$. Sessenta por cento dos participantes que responderam que uma gestante poderia consumir bebidas alcoólicas, desde que consideradas "fracas", são mulheres. Dos participantes que entendem que uma gestante não deveria consumir nenhuma quantidade de bebida alcoólica, 53,3\% são mulheres. Entre os participantes que são permissivos ao consumo de álcool pela gestante, o tipo de bebida mais permitido é a cerveja preta, escolhida por 32,1\% $(\mathrm{n}=17)$ dos participantes considerados permissivos; desses, 64,7\% são mulheres $(\mathrm{n}=11))$, embora deva ser restrita a uma só dose, conforme pensam $67,3 \%$ desses participantes permissivos. O vinho foi apontado como permitido por $24,5 \%$ dos participantes permissivos ao consumo de bebidas alcoólicas em ocasiões especiais. Entre as gestantes, $73 \%$ das participantes pensam que mulheres em sua condição não devem consumir bebidas alcoólicas, mesmo em ocasiões especiais. Nessa amostra, 16\% das gestantes são permissivas ao consumo de bebidas alcoólicas, desde que sejam aquelas consideradas "fracas", sendo eleita, por $45 \%$ das gestantes permissivas ao uso de álcool, a cerveja preta.

A mesma tendência foi observada quando os participantes eram perguntados a respeito do consumo de bebidas alcoólicas em ocasiões especiais por mulheres em fase de amamentação. Da população geral, 71,5\% dos participantes sustentam que uma mulher que está amamentando não deve consumir bebidas alcoólicas, mesmo em ocasiões especiais (52,1\% são mulheres); e $13 \%$ dos participantes pensam que a lactante pode consumir bebidas alcoólicas, desde que sejam aquelas consideradas "fracas". Entre as gestantes, 83,2\% pensam que uma mulher que está amamentando não deve consumir bebidas alcoólicas e 10,9\% são permissivas ao consumo de bebidas "fracas".

\section{Do consumo de bebidas alcoólicas em almoços de domingo}

Os participantes foram questionados quanto à possibilidade de uma gestante ou de uma mulher que está amamentando consumir bebidas alcoólicas aos domingos. Entre a amostra da população geral, 75,6\% dos participantes entendem que uma gestante não deve consumir álcool, mesmo que de forma restrita a um dia da semana $(53,1 \%$ mulheres $)$, enquanto $15,1 \%$ pensam que ela poderia beber, mas menos do que se não estivesse grávida (57,7\% mulheres). Quando é permitido à gestante consumir bebidas alcoólicas aos domingos, $63,6 \%$ pensam que o consumo deve ser restrito a apenas uma dose, sendo a cerveja preta a bebida escolhida por $28,3 \%(n=13)$ dos participantes permissivos. Quando considerada uma mulher que está amamentando, 80,6\% dos participantes entendem que não se deve consumir bebidas alcoólicas e 10,5\% entendem que, se a gestante pode beber, deve ser menos do que consumia antes de engravidar. Dos participantes permissivos ao consumo de bebidas alcoólicas, 51,4\% entendem que o consumo deve ficar restrito a uma dose e $27 \%$ a um só gole, e a bebida de eleição é, mais uma vez, a cerveja preta $(51,4 \%)$.
Entre as gestantes, $83,2 \%$ pensam que uma mulher em sua condição não deve consumir bebidas alcoólicas, ainda que de forma restrita a um dia na semana, e 9,6\% pensam que, se uma gestante pode beber, o tipo de bebida deve ser "fraco". Entre as gestantes permissivas, a metade pensa que a quantidade de bebida consumida deve se restringir a uma dose, e $28 \%$ a um gole. Já considerando a mulher que se encontra em fase de amamentação, 92\% das gestantes pensam que ela não deve consumir nenhuma quantidade de bebida alcoólica.

\section{Do consumo diário de bebidas alcoólicas}

Os participantes oriundos da população geral entendem, em sua maioria $(95,3 \%)$, que uma gestante não deve consumir bebidas alcoólicas diariamente (53,1\% mulheres). Dos participantes permissivos ao consumo de álcool durante a gestação, 41,7\% $(n=5)$ entendem que a cerveja preta pode ser consumida em uma dose $(50 \%)$ ou um gole $(50 \%)$. Nessa mesma amostra $(90,1 \%)$, se entende que mulheres em fase de amamentação não devem consumir bebidas alcoólicas diariamente; 4,7\% dos participantes entendem que elas podem consumir bebidas alcoólicas, desde que bebidas fracas, sendo a cerveja preta apontada por $62 \%$ dos participantes permissivos ao consumo de bebidas alcoólicas, durante a fase de amamentação, restritas a um gole ou uma dose (40\% dos participantes permissivos).

Entre as gestantes, 93\% pensam que uma mulher em sua condição não deve consumir bebidas alcoólicas, 93,4\% delas aplicando a mesma regra para mulheres em fase de amamentação.

Da amamentação: uma mulher que parou de beber durante a gravidez poderia voltar a beber durante a amamentação?

$\mathrm{Na}$ amostra oriunda da população geral, $85 \%$ dos participantes entendem que uma mulher que parou de beber durante a gestação não deveria voltar a consumir bebidas alcoólicas durante a amamentação. A amostra de gestantes não mostrou grande diferença, 93\% delas pensando o mesmo que a população geral quanto ao consumo de álcool, nessa fase da vida.

\section{Do fumo durante a gestação e a amamentação}

Perguntados a respeito da possibilidade de uma fumante continuar fumando durante a gestação, 92,4\% dos participantes advindos da amostra da população geral pensam que a gestante deveria se abster totalmente do consumo de cigarros, $4,7 \%$ entendem que ela até poderia fumar, mas deveria diminuir a quantidade consumida. Nessa mesma amostra, 87,8\% dos participantes entendem que uma mulher que parou de fumar, durante a gestação, não deveria voltar a fumar durante a amamentação.

Entre as gestantes, 94,2\% pensam que a fumante deve parar de fumar por ocasião da gravidez, e deve evitar tal hábito também durante a amamentação $(89,1 \%)$.

Do uso de medicação sem prescrição 
Os participantes da amostra oriunda da população geral $(91 \%)$ pensam que uma gestante não deve consumir remédios sem indicação médica, tais como antiinflamatórios, antidepressivos, antibióticos e moderadores de apetite, mesmo que o fizesse com frequência antes da gravidez. Esses participantes (92,4\%) também entendem que isso se estende a mulheres em fase de amamentação.

Entre as gestantes, 93,4\% entendem que uma mulher deve evitar a automedicação em sua condição, bem como na fase de amamentação $(91,2 \%)$.

\section{Do uso de drogas ilegais}

Os participantes que representam a população geral $(98,3 \%)$ entendem que uma mulher que usa drogas ilegais deve parar de consumi-las ao engravidar, o mesmo se aplicando à mulher em fase de amamentação (98,8\%).

A amostra de gestantes mostrou a mesma tendência (100\%), o mesmo se estendendo ao que pensam em relação à lactante $(100 \%)$.

\section{Discrepância}

Um aspecto bastante interessante do presente estudo diz respeito à identificação da discrepância na atitude das gestantes quanto ao consumo de cigarros durante a gravidez. Enquanto $94,2 \%$ das gestantes entrevistadas revelaram atitude de restrição ao uso de cigarros $(5,8 \%$ foram permissivas), $17,5 \%$ delas utilizaram alguma quantidade do produto (sendo que 38,9\% das fumantes admitiram ter fumado até meia carteira de cigarros por dia), demonstrando que, pelo menos, $11,7 \%$ das gestantes fumaram admitindo os efeitos deletérios do uso de cigarros durante a gestação.

\section{Diferenças entre resultados das amostras}

Para se comparar as tendências de resultados entre as duas amostras abordadas, esses foram sumarizados nas Tabelas 1 e 2. Os resultados estão descritos em porcentagens, sendo o cenário descrito na primeira linha da tabela, associados à segunda linha, que envolve a possibilidade de uma gestante ou de que uma lactante utilize a substância. Na terceira linha estão descritas as origens dos resultados, se oriundos da amostra que representa a população geral ou gestantes e, finalmente, na quarta linha, os resultados.

Tabela 1 - Atitudes de restrição ao consumo de álcool na gravidez e na amamentação (\%)

\begin{tabular}{|c|c|c|c|c|c|c|c|c|c|c|c|c|}
\hline \multirow{3}{*}{$\begin{array}{l}\text { Cenário } \\
\text { Amostra }\end{array}$} & \multicolumn{4}{|c|}{$\begin{array}{c}\text { Restrição ao consumo de álcool em } \\
\text { ocasiões especiais }\end{array}$} & \multicolumn{4}{|c|}{$\begin{array}{l}\text { Restrição ao consumo de álcool aos } \\
\text { domingos }\end{array}$} & \multicolumn{4}{|c|}{ Restrição ao consumo diário de álcool } \\
\hline & \multicolumn{2}{|c|}{ Gravidez } & \multicolumn{2}{|c|}{ Amamentação } & \multicolumn{2}{|c|}{ Gravidez } & \multicolumn{2}{|c|}{ Amamentação } & \multicolumn{2}{|c|}{ Gravidez } & \multicolumn{2}{|c|}{ Amamentação } \\
\hline & Geral & Gestantes & Geral & Gestantes & Geral & Gestantes & Geral & Gestantes & Geral & Gestantes & Geral & Gestantes \\
\hline Resultados & 71,8 & 73 & 71,5 & 83,2 & 75,6 & 83,2 & 80,6 & 92 & 95,3 & 93 & 90,1 & 93,4 \\
\hline
\end{tabular}

Tabela 2 - Atitudes de restrição ao consumo de outras SPAs na gravidez e na amamentação (\%)

\begin{tabular}{|c|c|c|c|c|c|c|c|c|c|c|c|c|}
\hline \multirow{3}{*}{$\begin{array}{l}\text { Cenário } \\
\text { Amostra }\end{array}$} & \multicolumn{4}{|c|}{ Restrição ao consumo de cigarros } & \multicolumn{4}{|c|}{$\begin{array}{l}\text { Restrição ao consumo de remédios sem } \\
\text { prescrição }\end{array}$} & \multicolumn{4}{|c|}{ Restrição ao consumo de drogas ilegais } \\
\hline & \multicolumn{2}{|c|}{ Gravidez } & \multicolumn{2}{|c|}{ Amamentação } & \multicolumn{2}{|c|}{ Gravidez } & \multicolumn{2}{|c|}{ Amamentação } & \multicolumn{2}{|c|}{ Gravidez } & \multicolumn{2}{|c|}{ Amamentação } \\
\hline & Geral & Gestantes & Geral & Gestantes & Geral & Gestantes & Geral & Gestantes & Geral & Gestantes & Geral & Gestantes \\
\hline Resultados & 92,4 & 94,2 & 87,8 & 89,1 & 91 & 93,4 & 92,4 & 91,2 & 98,3 & 100 & 98,8 & 100 \\
\hline
\end{tabular}

Como se pode observar, as tendências de resultados são bastante parecidas, por vezes a população geral manifestando maior número de atitudes de restrição, por vezes as gestantes manifestando maior número de atitudes de restrição, com poucas diferenças.

\section{Discussão}

O objetivo do presente estudo foi conhecer as atitudes em relação ao consumo de SPAs, durante a gestação e a amamentação, em uma amostra de gestantes e da população geral, se de restrição ou de permissividade. Como se pode acompanhar, pelos resultados, existe tendência geral, tanto na amostra oriunda da população geral quanto na amostra de gestantes, de restrição ao consumo de SPAs, durante a gestação e a amamentação. Os índices de respostas tendem à conclusão que não se deve usar SPAs durante tais períodos da vida, e mesmo aqueles que são permissivos entendem que o consumo deve ser diminuído no caso de todas as SPAs e medicações sem prescrição e, além disso, substituído, no caso do álcool, por bebidas de menor teor alcoólico. Os resultados são coerentes com o encontrado em um estudo qualitativo, onde se verificou que a maioria das gestantes adolescentes, que compôs esse estudo, classificou o consumo de álcool durante a gestação como "atitude inconsequente", já que tal hábito provoca prejuízos pré e pós-natais ao desenvolvimento do bebê(10).

Em relação ao consumo de bebidas alcoólicas, as gestantes do presente estudo tenderam a apresentar mais restrição em relação ao consumo no período da amamentação, talvez mais impactadas pela consequência direta e concreta de seus hábitos sobre a alimentação do filho. Além disso, o uso de SPAs durante a amamentação estava sendo avaliado como uma situação ainda não 
vivenciada, o que pode ter facilitado a restrição maior. Também há tendência geral, tanto da amostra da população geral quanto das gestantes, a maior restrição ao consumo de cigarros, de medicações sem prescrição e de drogas ilegais durante tais períodos do que o são em relação ao consumo eventual de álcool (em situações especiais e aos domingos). Os índices de restrição ao consumo diário de álcool, no entanto, se assemelham aos da restrição ao consumo das outras drogas.

Tais dados sugeriram que os participantes do presente estudo estão informados a respeito das consequências negativas do uso de SPAs e de medicações sem prescrição durante tais períodos da vida. Esses resultados são diferentes daqueles apresentados em estudo desenvolvido, no ano 1996, quando apenas $37 \%$ das gestantes estavam informadas a respeito do uso de drogas de abuso ${ }^{(11)}$ e dos resultados de um estudo apresentado em 2006, no qual é mostrado que $95 \%$ das gestantes admitiram não conhecer informações formais a respeito do uso de álcool durante a gestação, embora demonstrassem concepções sobre as consequências prejudiciais do consumo do álcool no feto, consideradas pelos autores experiências provenientes do senso comum e da experiência empírica ${ }^{(12)}$.

Essas diferenças podem ser devidas ao modo como o conhecimento sobre o uso de SPAs foi investigado nos três estudos. No estudo de $1996^{(11)}$, as gestantes eram indagadas a respeito de quais informações tinham sobre o uso de drogas durante a gestação, enquanto no presente estudo, isso não foi feito diretamente, mas através da descrição de cenários a serem julgados pelos participantes. Da mesma forma, no estudo de 2006, foram apresentadas duas personagens gestantes, cujas atitudes seriam julgadas por seus participantes ${ }^{(12)}$. Também diferenças devidas à passagem do tempo (por exemplo, maior acesso à informação hoje) e às características regionais (informações oferecidas por instituições de educação e saúde, por exemplo) poderiam explicar esses resultados. Uma interpretação mais otimista seria de que campanhas, tais como a antitabagismo, articuladas pelo Ministério da Saúde ${ }^{(7)}$, tenham aumentado a informação sobre o uso de SPAs. Se, por um lado, é possível que a informação tenha aumentado, por outro lado, estudos têm demonstrado que o uso de SPAs entre mulheres e durante a gestação continua crescendo. Entre grupos de adolescentes, é possível encontrar gestantes que são estimuladas a consumir bebidas alcoólicas por seus pares, ou que negligenciam e minimizam as informações sobre as consequências desse consumo para o desenvolvimento de seus filhos, para, consumindo, se sentirem mais próximas de seus pares e na plenitude das experiências oferecidas por seu período de desenvolvimento ${ }^{(10)}$. Em contrapartida, os resultados, em especial da amostra da população geral, mostram que campanhas de cessação do uso de SPAs, durante a gestação e a lactação, podem ter como alvo não apenas gestantes e mães, mas pessoas próximas a elas, com ênfase especial em amigas confidentes que, estranhamente ou não, têm mais influência sobre o comportamento da gestante do que seus parceiros ${ }^{(3)}$.

Em oposição aos dados epidemiológicos de utilização de SPAs durante a gestação(9,13-17), é bastante positivo o fato de que os índices de atitudes de restrição ao consumo de SPAs durante a gestação tenham se mostrado altos. Entretanto, fica evidente a discrepância de atitude na amostra de gestantes estudadas, o que pode ser importante objeto de estudo e possível alvo dentro de campanhas de prevenção ao uso de SPAs, durante a gestação e a amamentação. Essa discrepância pode ser um alerta, pois, gestantes fumantes, diferentemente daquelas que tentam parar de fumar e das não fumantes, foram as que avaliaram menos negativamente o hábito de fumar na gestação em um estudo de tomada de decisão ${ }^{(18)}$.

Programas de estimulação à amamentação e cursos de gestantes podem contribuir para diminuir o uso de SPAs. A probabilidade de fumar durante a gestação diminui se, antes da gestação, a mulher decide que amamentará seu bebê por mais de quatro meses ${ }^{(19)}$. Em contrapartida, não comparecer a cursos de gestantes e não intencionar amamentar durante mais que quatro meses, bem como ter parceiros fumantes ${ }^{(10,15,17)}$ e ter consumido bebidas alcoólicas antes da gestação parecem ser fatores significativos para o uso de cigarros.

Atitude, portanto, não deve ser tratada como um conceito central para a modificação do uso de SPAs, durante a gestação e a amamentação, pois outros fatores podem ser determinantes desse uso. É possível que o uso de SPAs durante esses períodos esteja também associado à presença de transtornos mentais. Por exemplo, já foi verificado que quase metade das gestantes tabagistas $(45,1 \%)$ apresentava critérios para o diagnóstico de, pelo menos, um transtorno mental, sendo que entre as gestantes dependentes do tabaco (12,4\% das fumantes), mais da metade $(57,5 \%)$ apresentava critérios para um segundo transtorno mental ${ }^{(20)}$. Comportamentos de autocuidados, que incluem o acato de orientações médicas, estão diminuídos quando existe um transtorno mental ${ }^{(21)}$.

O tratamento precoce de transtornos mentais em períodos pré e pós-natal é recomendação da Organização Mundial de Saúde, já que beneficia não apenas a saúde mental da mãe, mas favorece o desenvolvimento saudável da criança ${ }^{(21)}$. Se atitude corresponde à combinação entre crenças, sentimentos e, seu produto final, os comportamentos, destaca-se a discrepância entre crença e comportamento de uso de SPAs, durante a gestação e a amamentação. Tal discrepância remete ao fato de que atitudes nem sempre permitem prever o comportamento, embora eventualmente o permitam, sendo que a questão preponderante não é se as atitudes predizem o comportamento, mas quando as atitudes predizem o comportamento, o que depende de características individuais, das situações e da atitude em si, o que empobrece o poder preditivo da atitude. Além disso, uma atitude pode ser considerada ambivalente, se avaliada tanto positiva quanto negativamente pelo mesmo indivíduo, sendo essa ambivalência um estado desconfortável que, geralmente, depende do contexto e tende à mudança ${ }^{(4)}$.

O comportamento de uso de SPAs, durante a gestação e a amamentação, é geralmente acessado através de medidas de autorrelato. Essas medidas podem não ser 
confiáveis justamente pela discrepância entre a crença e comportamento, além do constrangimento gerado pela forte pressão social e o reconhecimento dos malefícios do tabagismo sobre a saúde materna e fetal, o que faz com que grande parte das gestantes não preste informações verídicas a respeito do seu hábito de fumar ${ }^{(1)}$ ou de consumir outras substâncias.

Tais diferenças já foram demonstradas em estudos que compararam o autorrelato de gestantes com marcadores biológicos. Embora em índices não significativos para a invalidação dos autorrelatos, parte das gestantes que se declararam não fumantes apresentou marcadores biológicos positivos para o uso de tabaco (23\% das gestantes declararam ser tabagistas, enquanto $25 \%$ delas apresentaram marcadores biológicos de tabagismo), no caso, a partir de amostras de $\mathrm{CO}^{(22)}$. Em gestantes adolescentes brasileiras, verificou-se, através da análise dos fios de cabelo, que $6 \%$ utilizaram cocaína ou maconha no terceiro trimestre de gravidez, $4 \%$ consumiram unicamente maconha, $1,7 \%$ consumiram unicamente cocaína e 0,3\% utilizaram ambas as drogas, sendo que nenhuma delas reportou uso de substâncias em um questionário utilizado para investigar o uso de SPAs durante a gestação(23).

Mesmo com o conhecimento de todas as consequências das SPAs, muitas vezes, mesmo equipes médicas bem treinadas, por diversos motivos, não estão atentas para advertir a respeito da necessidade de abandonar o uso de SPAs, embora concordem fortemente que gestantes deveriam parar de utilizar qualquer substância ${ }^{(24)}$, o que demonstra a dificuldade em transpor atitudes a intervenções práticas. Crenças pessoais sobre o tabagismo como estratégia para lidar com o estresse também são empecilho para intervenções bem-sucedidas. A crença de que gestantes adultas não devem ser forçadas a tomar decisões sobre questões pertinentes a sua saúde, tais como parar de fumar, e pelo medo por parte da equipe de saúde que tal sentimento possa afastar as gestantes dos cuidados médicos, também pode ser considerada uma barreira à cessação do uso de $\operatorname{cigarros}^{(3)}$, bem como o constrangimento por parte de pares, no caso de adolescentes gestantes usuárias de álcool ${ }^{(10)}$.

\section{Conclusões}

Os importantes índices de atitudes de restrição ao consumo de SPAs, durante a gestação e a amamentação, presentes tanto na amostra oriunda da população geral quanto entre gestantes, demonstram que os participantes do presente estudo estão informados a respeito das consequências negativas do uso de SPAs e de medicações sem prescrição durante tais períodos da vida. Os resultados permitem concluir também que campanhas que tenham como alvo as atitudes de gestantes e mães podem ser efetivas na prevenção do uso de SPAs, durante a gestação e a lactação. Os resultados, em especial da amostra da população geral, mostram que essas campanhas podem ter como alvo não apenas gestantes e mães, mas pessoas próximas a elas. Assim, é favorável estimular que pessoas relevantes às gestantes opinem a respeito do uso de SPAs e se posicionem em relação à saúde da gestante e de seu filho.

Além disso, os resultados do presente estudo possibilitam concluir que as atitudes de restrição ou de permissividade em relação ao consumo de SPAs, durante a gravidez e a amamentação, não são exatamente consistentes com os índices de uso de SPAs durante a gestação. Se, no presente estudo, que utilizou uma medida de autorrelato, identificou-se que $11,7 \%$ das gestantes consumiram cigarros mesmo conhecendo os efeitos deletérios do mesmo sobre a saúde do filho, suspeita-se que percentual maior de gestantes o tenha consumido sem relatar, considerando também os dados fornecidos pela literatura científica explorada no que diz respeito a outras drogas.

O presente estudo não permite concluir, no entanto, porque o uso de SPAs durante a gestação e a lactação associado a uma atitude de restrição ocorre. O valor funcional das atitudes e a influência que elas exercem nos julgamentos e comportamentos podem variar e, além disso, como se viu, uma atitude pode ser ambivalente e contexto dependente, sendo que essa ambivalência pode ser uma hipótese para o uso de SPAs associado à atitude de restrição, somente a ser confirmada a partir de estudos futuros que explorem tal relação. Cabe destacar que a discrepância entre a atitude e o comportamento não é fenômeno exclusivo do uso de SPAs durante a gestação, sendo também observado, por exemplo, em estudos de atitudes em relação a uso de preservativos ou à alimentação saudável. Assim, nem todas as atitudes são iguais ou podem ser avaliadas da mesma forma.

É sugerido o desenvolvimento de novos estudos que permitam entender a relação entre atitudes e uso de SPAs durante a gestação e a amamentação, abordando a dependência química específica, os fatores estressantes que podem desencadeá-la, a presença de comorbidades, a percepção dos riscos envolvidos no consumo, e de crenças favoráveis ao uso de SPAs durante tais períodos em contextos específicos.

Introduzir ações que façam alguma diferença para mudar o comportamento torna a atitude mais coerente com ideais de saúde. Campanhas de prevenção e de cessação do uso de SPAs durante a gestação e a lactação que tenham como alvo as atitudes, somadas a outras medidas de eficácia comprovada, tendem a aumentar as taxas de sucesso, diminuindo a ambivalência, reforçando atitudes positivas e pró-saúde.

\section{Referências}

1. Leopércio W, Gigliotti A. Tabagismo e suas pecliaridades durante a gestação: uma revisão crítica. J Bras Pneumol. 2004;30(2):176-85.

2. Ockene JK, Ma Y, Zapka JG, Pbert LA, Valentine G. K, Stoddard AM. Spontaneous cessation of smoking and alcohol use among low-income pregnant women. Am J Prev Med. 2002;23(3):150-9.

3. Dunn CL, Pirie PL, Hellerstedt WL. Lay advice on 
alcohol and tobacco during pregnancy. Health Care Women In. 2004;25:55-75.

4. Fazio RH, Olson M. Attitudes: foundations, functions and consequences. In: Hogg MA, Cooper J, editors. Sage Handbook of Social Psychology. London: Sage; 2003.

5. Bull L, Burke R, Walsh S, Whitehead E. Social attitudes towards smoking in pregnancy in East Surrey: a qualitative study of smokers, former smokers and non-smokers. J Neonat Nurs. 2007;13:100-6.

6. Meneguin AMPL. O imaginário da drogadição: uma análise das campanhas antidrogas. In: Comunicação I-SBdEId, editor. $25^{\circ}$ Congresso Brasileiro de Ciências da Comunicação; 2002; Salvador/BA; 2002.

7. Cavalcante T. O controle do tabagismo no Brasil: avanços e desafios. Rev Psiq Clín. 2005;32(5):283-300.

8. Nascimento BEM, Oliveira L, Vieira AS, Joffily M, Gleiser S, Pereira MG, et al. Avoidance of smoking: the impact of warning labels. Tobbaco Control. 2008;17:4059.

9. Ihlen BM, Amundsen A, Tronnes L. Reduced alcohol use in pregnancy and changed attitudes in the population. Addiction. 1993;88:389-94.

10. Costa TS, Vasconcelos TC, Sousa LB, Bezerra CP, Miranda FAN, Alves SGS. Percepções de adolescentes grávidas acerca do consumo de álcool durante o período gestacional. SMAD, Rev Eletr Saúde Mental, Álcool e Drogas. [periódico na internet]. 2010;6(1).[acesso 3 julho 2010]. Disponível em: http://www2.eerp.usp.br/resmad/ artigos/SMADv6n1a02.pdf

11. Costa MTZ, Quintal VS, Haro FMB, Picchi M, Salgado MVS, Okay Y, et al. Drogas de abuso na gestação: as orientações no pré-natal são suficientes? Pediatria. (São Paulo). 1998;20(4):317-22.

12. Fiorentin CF, Vargas D. O uso de álcool entre gestantes e seus conhecimentos sobre os efeitos do álcool no feto. SMAD, Rev Eletr Saúde Mental, Álcool e Drogas. [periódico na internet]. 2006;2(2). [acesso 1 julho 2010]. Disponível em: http://www2.eerp.usp.br/resmad/ artigos/2006v2n2a05.pdf

13. Grangé G, Vayssiere C, Borgne A, Ouazana A, L'Huillier J, Valensi P, et al. Description of tobacco addiction in pregnant women. Eur J Obstet Gynecol R B. 2005;120(2):146-51.

14. Horta BL, Victora CG, Barros FC, Santos IS, Menezes AMB. Tabagismo em gestantes de área urbana da região sul do Brasil, 1982 e 1993. Rev Saúde Pública.
1997;31(3):247-53.

15. Kroeff LS, Mengue SS, Schmidt MI, Duncan BB, Favaretto ALF, Nucci BN. Fatores associados ao fumo em gestantes avaliadas em cidades brasileiras. Rev Saúde Pública. 2004;38(2):261-7.

16. Lambers DS, Clark KE. The maternal and fetal physiologic effects of nicotine. Semin Perinatol. 1996;20(2):115-26.

17. Moraes CL, Reichenhein ME. Rastreamento de uso de álcool por gestantes de serviços públicos de saúde do Rio de Janeiro. Rev Saúde Pública. 2007;41(5):695-703.

18. Ortendahl M. Smoking as a decision among pregnant and non-pregnant women. Addict Behav. 2006;31(10):1806-19.

19. Giglia RC, Binns CW, Alfonso HS, Zhan Y. Which mothers smoke before, during and after pregnancy? Public Health. 2007 Dec;121(12):942-9. Epub 2007 Jun 18.

20. Goodwin RD, Keyes K, Simuro N. Mental disorders and nicotine dependence among pregnant women in the Unites States. Obstet Gynecol. 2007;109(4):875-83.

21. WHO-UNFPA. Mental health aspects of women's reproductive health: A global review of the literature. Geneva: World Health Organization; 2009.

22. Campbell E, Sanson-Fisher R, Walsh R. Smoking status in pregnant women: assessment of self-report against carbon monoxide (CO). Addict Behav. 2001;26:1-9.

23. Bessa MA, Mitsuhiro SS, Chalem E, Barros MM, Guinsburg R, Laranjeira R. Underreporting of use of cocaine and marijuana during the third trimester of gestation among pregnant adolescents. Addict Behav. 2010;35(3):266-9.

24. Condliffe L, McEwen A, West R. The attitude of maternity staff to, and smoking cessation interventions with, childbearing women in London. Midwifery. 2005;21(3):233-40.

\section{Como citar este artigo:}

Matta A, Soares LV, Bizarro L. Atitudes de gestantes e da população geral quanto ao uso de substâncias durante a gestação. SMAD, Rev. Eletrônica Saúde Mental Álcool Drog. (Ed. port.). set.-dez. 2011 [acesso:

47. Disponível em:

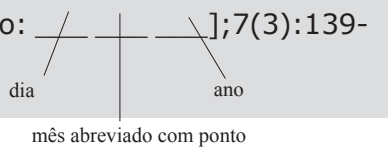

